\title{
An interface compatibility/equilibrium mechanism for delamination fracture in aluminum-lithium alloys
}

\author{
M. C. Messner ${ }^{\mathrm{a}}$, A. J. Beaudoin ${ }^{\mathrm{b}}$, R. H. Dodds, Jr. ${ }^{\mathrm{a}, *}$ \\ ${ }^{a}$ Department of Civil and Environmental Engineering, 2113 NCEL, MC 250, University of Illinois at Urbana-Champaign, \\ 205 N. Mathews Avenue, Urbana, IL 61801, USA \\ ${ }^{b}$ Department of Mechanical Science and Engineering, 354 MEB, MC 244, University of Illinois at Urbana-Champaign, 1204 \\ Green Street, Urbana, IL 61801, USA
}

\begin{abstract}
This work describes a mechanism for the initiation of delamination cracks in Al-Li alloys based on the soft/stiff character of adjacent grains. Small-scale-yielding, crystal plasticity simulations of divider grain configurations (L-T) reveal an elevated mean stress on grain boundaries. This mean stress increase drives a sharp localization of the Rice-Tracey parameter to the grain boundaries - elevation of the RT parameter indicates favorable conditions for void growth and triggering of delamination cracking, in agreement with the fractography of Ritchie and co-workers. Our simulation results and available experimental evidence indicate delamination initiates typically between soft/stiff grain pairs, often Bs (Bunge-convention Euler angles $\left.\phi_{1}=131^{\circ}, \Phi=83^{\circ}, \phi_{2}=307^{\circ}\right)$ or $\mathrm{S}\left(\phi_{1}=233^{\circ}, \Phi=151^{\circ}, \phi_{2}=105^{\circ}\right)$ orientations. The crystal plasticity results and a simple model of a soft/stiff material interface show that mean stress accumulation is a consequence of the mechanics of such an interface, and not necessarily tied to material inhomogeneities near the GBs (such as precipitate free zones).

Keywords: Delamination; Intergranular fracture; Aluminum alloys; Micromechanics; Aerospace vehicles
\end{abstract}

\section{Introduction}

Replacement of conventional aerospace aluminum alloys with aluminum-lithium alloys in high-performance structures has the potential to generate significant weight- and cost-savings. A lack of predictive tools for the initiation and propagation of intergranular delamination cracks in modern Al-Li alloys often necessitates extensive testing to establish the delamination behavior of specific components [1]. Delamination occasionally triggers fracture in other metals alloys as well. Some steels, such as 3CR12 and X70, fail by delamination under certain loading conditions; conventional aluminum alloys (7050) also delaminate, particularly in fatigue loading [2-4]. The delamination behavior in all these materials depends strongly on loading and crack orientation relative to the material rolling axes. This work builds on previous experimental and computational

\footnotetext{
${ }^{*}$ Corresponding author. Tel.: +1-217-390-0034

Email addresses: mmessne2@illinois.edu (M. C. Messner), abeaudoi@illinois.edu (A. J. Beaudoin), rdodds@illinois.edu (R. H. Dodds, Jr.)
} 


\begin{tabular}{|c|c|}
\hline \multicolumn{2}{|c|}{ Nomenclature } \\
\hline\|\|$_{F}$ & Frobenius norm \\
\hline$\alpha$ & Nye tensor of necessary dislocation density \\
\hline$\dot{\gamma}^{(s)}$ & Slip rate along slip system $s$ \\
\hline$\dot{\gamma}_{0}$ & Reference strain rate \\
\hline$\Delta \varepsilon$ & Compatibility strains \\
\hline$\Delta \sigma$ & Equilibrium stresses \\
\hline$\varepsilon^{\mu}$ & Microscale strain field \\
\hline$\dot{\vec{\varepsilon}}^{p}$ & Equivalent plastic strain rate $\dot{\bar{\varepsilon}}^{p}=\sqrt{\frac{2}{3} \dot{\varepsilon}_{i j}^{p} \dot{\varepsilon}_{i j}^{p}}$ \\
\hline$\nu$ & Poisson's ratio \\
\hline$\sigma^{\mu}$ & Microscale stress field \\
\hline$\sigma_{m}$ & Mean stress $\sigma_{m}=1 / 3 \operatorname{tr}(\sigma)$ \\
\hline$\tilde{\tau}$ & Slip system strength or backstress \\
\hline$\tau$ & Kirchhoff stress \\
\hline$\phi_{1}, \Theta, \phi_{2}$ & Bunge convention Euler angles \\
\hline $2 \mathrm{D}$ & Two dimensional \\
\hline $3 \mathrm{D}$ & Three dimensional \\
\hline$B$ & Model thickness \\
\hline $\mathbf{b}^{(s)}$ & Slip direction for slip system $s$ \\
\hline $\mathrm{CP}$ & Crystal plasticity constitutive model \\
\hline $\mathrm{C}(\mathrm{T})$ & Compact-Tension fracture specimen \\
\hline D & Macroscopic rate of deformation tensor \\
\hline$E$ & Young's modulus \\
\hline $\mathbf{F}$ & Deformation gradient \\
\hline $\mathbf{F}^{e}$ & Elastic portion of deformation gradient, $\mathbf{F}=\mathbf{F}^{e} \mathbf{F}^{p}$ \\
\hline $\mathbf{F}^{p}$ & Plastic portion of deformation gradient, $\mathbf{F}=\mathbf{F}^{e} \mathbf{F}^{p}$ \\
\hline$(L, T, S)$ & Material rolling coordinate system: longitudinal, transverse, and short directions \\
\hline $\mathrm{M}(\mathrm{T})$ & Middle-cracked tension specimen \\
\hline $\mathbf{n}^{(s)}$ & Slip normal for slip system $s$ \\
\hline$n$ & Rate exponent \\
\hline$n_{\text {slip }}$ & Number of slip systems in model \\
\hline$R$ & Model radius \\
\hline $\mathbf{R}^{e}$ & Elastic rotation, $\mathbf{F}^{e}=\mathbf{V}^{e} \mathbf{R}^{e}$ \\
\hline RT & Rice-Tracey void growth parameter \\
\hline SSY & Small scale yielding \\
\hline & Grain thickness \\
\hline$(x, y, z)$ & Cartesian crack-tip coordinate system \\
\hline
\end{tabular}

studies of delamination in Al-Li alloys to describe a plausible mechanism for delamination events based on the existence of soft/stiff grain interfaces.

The flat, pancake-shaped grain structure of rolled Al-Li plate material influences the plane and direction of delamination crack growth - delaminations follow the wide L-T grain boundaries, occasionally crossing from one L-T boundary to another at or near the end of a grain $[5,6]$. Figure 1 shows the standard series of primary crack/delamination crack configurations relative to the specimen rolling directions (see appendix for additional details). This figure also shows a micrograph of a slice of material ahead of the primary crack plane in a divider configuration taken from an arrested fracture test of a side-grooved $\mathrm{C}(\mathrm{T})$ 
specimen (sectioned and polished to reveal delamination cracks). The micrograph shows small, vertical divider delaminations developing ahead of the primary crack plane. Previous research describes the effects these divider delaminations, and delamination cracks in other configurations, have on fracture and fatigue behavior of the primary crack. Rao and Ritchie [7-14] conducted some of the earliest work on the effect of delamination cracks on primary crack growth in second-generation Al-Li alloys. For most experimental configurations, they concluded that delamination cracking increases the apparent fracture toughness of the material via deflection and shielding of the primary crack. They observed an increase in delamination fracture events relative to primary crack growth at cryogenic temperatures, leading to an increase in overall toughness at lower temperatures. Furthermore, the fracture morphology of delaminated surfaces revealed voids and coalesced voids as evidence for delamination formation and extension by a void-growth driven process. These findings remain true with modern, third generation Al-Li alloys [15, 16]. Macroscale fracture simulations with prescribed delaminations also demonstrate increased primary crack toughness, as the delamination cracks lead to loss of constraint ahead of the primary crack front and reduced driving forces on the primary crack plane $[17,18]$.

These previous studies examined the consequence of pre-existing delamination cracks on the fracture behavior of primary cracks. Less established are the actual mechanism and conditions leading to the onset of delaminations very near the front of the primary crack. This work focuses on the mechanics of soft/stiff grain boundaries that appear to trigger delamination cracking. These soft/stiff boundaries separate grains with distinctly different orientations that deform with very different stress states - one grain remains essentially linear-elastic while the other deforms plastically. Recent experiments by Beaudoin and colleagues demonstrate the existence of such grain boundaries and suggest a link to the onset of delamination [19-21].

Mesoscale-type models [22] of delamination-prone grain boundaries under the loading produced by a primary crack provide insight into the highly-local fields driving fracture and potential failure mechanisms that develop on these soft/stiff boundaries. Material models adopting crystal plasticity capture the mesoscale constitutive response of metals undergoing plastic deformation [23, 24]. McDonald and Beaudoin suggest such mesoscale resolution is sufficient to predict the stress states leading to delamination [25]. We have previously modeled [26] the arrestor configuration (Fig. 1) with a crystal plasticity, finite-element framework and concluded that certain Bs and Bs/Bs grain pairs (Bunge-convention Euler angles $\phi_{1}=131^{\circ}, \Phi=83^{\circ}$, $\left.\phi_{2}=307^{\circ}\right) / \mathrm{S}\left(\phi_{1}=233^{\circ}, \Phi=151^{\circ}, \phi_{2}=105^{\circ}\right)$, found to delaminate in experiments, produce primary crack shielding. This work considers a different (divider) material configuration and develops a simple description of soft/stiff interface mechanics that leads to elevated mean stress and plastic void growth.

Delamination in Al-Li shares similarities with delamination in a laminar composite, albeit on a smaller representative length-scale. Sugimura et al., for example, modeled a crack near a soft/stiff material interface; that work motived by layered, bimetallic composites [27]. They concluded the soft/stiff interface either shields or amplifies the stress concentration at the primary crack, depending on the orientation of the material (soft/stiff or stiff/soft). The direct analogy to Al-Li is an arrestor delamination developing over a 


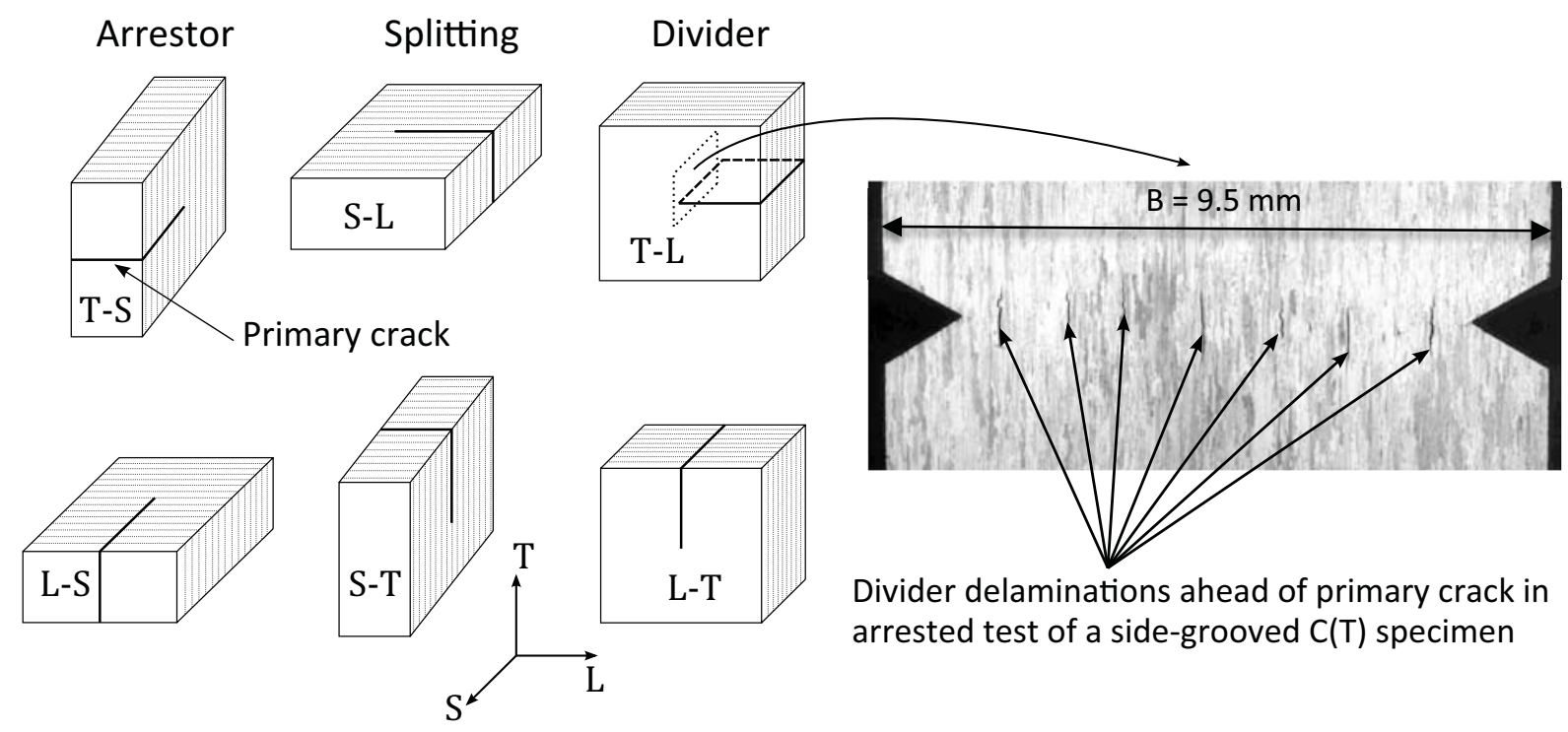

Figure 1: Standard primary-delamination crack configurations. This study examines divider configurations, in particular an L-T configuration. The micrograph is from a side-groove $\mathrm{C}(\mathrm{T})$ specimen, $\mathrm{T}$-L, with the test interrupted before final fracture. Sectioning/polishing reveal the divider delaminations forming in the region of high stress triaxiality ahead of the primary crack (reproduced from [18]).

soft/stiff grain boundary, a comparison explored in previous work [26]. In related work, Roy Chowdhury and Narasimhan modeled fracture in a polymeric adhesive layer joining two linear-elastic adherends [28]. They found the plastic zone radius and triaxiality ahead of a crack in the adhesive layer are strongly influenced by the pressure sensitivity and thickness of the adhesive layer, due to the high constraint provided by the surrounding stiff, elastic layers. Work by Lin et al. [29] and others highlighted the importance of increased triaxiality found in a soft, ductile layer constrained by stiff, elastic substrates. They focused on the impact of high triaxiality on propagating ductile, void-driven cracks.

This study explores the similarities between delamination in Al-Li and delamination in a laminar composite with interspersed soft and stiff layers. In laminar composites the stiff and soft layers are different materials, whereas in Al-Li the layers are pancake-shaped grains with different crystallographic orientations but similar elastic and slip system hardening properties. The orientation of a grain, relative to the remote loading near the primary crack, causes soft or stiff behavior compared to its neighbors. In CP simulations of alternating, layered L-T grain structures under the action of a Mode I primary crack, this effect is most pronounced on Bs (stiff)/S (soft) grain boundaries, in agreement with prior experimental observations and numerical calculations performed by Beaudoin et al. [30] for uniaxial tension. Furthermore, these soft/stiff, $\mathrm{S} /$ Bs grain boundaries develop elevated mean stress, compared to the macroscale crack-front fields, which drives increased void-growth rates. A simplified model proposed here treats the soft/stiff grains in a linearized sense as elastic materials with differing stiffness; the model predicts the mean stress elevation and that it is a consequence of interface equilibrium and compatibility requirements. 
Section 2 describes a small-scale-yielding, computational model for fracture in the L-T configuration that demonstrates soft/stiff mean stress concentration on Bs/S grain boundaries. The section also describes the gradient-enhanced CP model representing the mesoscale deformation of Al-Li grains and summarizes some relevant experimental observations from previous work. Section 3 describes results of the CP simulations; Section 4 discusses those results and develops the simplified model of a soft/stiff interface. Finally, Section 5 summarizes the conclusions of the paper and outlines future work to extend the simplified model for engineering-scale computations. An appendix provides further details on grain-scale plasticity, the slipsystem hardening model, and summarizes the CP model material properties.

\section{Materials and Methods}

\subsection{Experimental data}

Experimental observations [19-21] reveal the existence of soft and stiff grain orientations under the action of a macroscale primary crack - a soft grain has lower stresses and, in general, more plastic deformation than a stiff grain.

Indirect measurements of soft/stiff grains include those of Tayon et al., Hernquist, and Crooks et al. [20, 21, 31]. Tayon et al. and Hernquist calculated the Taylor factor of orientations on each side of a delamination using stresses thought to contribute towards the delamination. They found delaminations developed typically on GBs between grains with high and low Taylor factors. The Taylor factor correlates inversely to the amount of slip; low Taylor factor grains are soft, while high Taylor factor grains are stiff. The soft/stiff grain pairs were typically Bs or S variants. Crooks et al. measured the hardness of grains on each side of a delamination via nanoindentation. They found delamination occured typically between soft grains (low strain energy) and stiff grains (high strain energy). Furthermore, they observed this difference between soft and stiff grains occured only very near GBs - the bulk of the grains had approximately equal hardness.

Beaudoin et al. found soft/stiff grains in situ via high-energy diffraction microscopy (HEDM) in samples of 2195-T8 Al-Li. They used HEDM measurements to estimate the lattice strains and stresses in three distinct grains in a microtension specimen under uniaxial extension. One of the three grains deformed at much higher stress levels than the other two - a stiff grain. The macroscale stress state in these experiments was uniaxial, not the severe triaxial stresses expected along a crack front. The existence of soft and stiff grains very close together supports the hypothesis of soft/stiff boundaries triggering delamination (all three grains within $145 \mu \mathrm{m}$ in the $\mathrm{N}$-direction, and quite possibly adjacent, considering the average grain thickness).

\subsection{SSY model of divider delamination}

Based on these experimental observations and our prior work, an understanding of the driving conditions for divider delamination in $\mathrm{Al}-\mathrm{Li}$ becomes focused on the mesoscale response near the boundaries of soft/stiff neighboring grain pairs. This study considers a Bs orientation and a S orientation (see the Appendix for additional discussion of crystallographic orientations in metal alloys). This orientation pair delaminated 
in an experiment conducted by Beaudoin et al. [19]. Figure 2 shows the computational model used here. In the $x y$ plane, this is a typical small-scale yielding model with an initially sharp crack opening in the $y$-direction. The FE mesh and loading are symmetric about the $y=0$ plane. Alternating layers of Bs and $\mathrm{S}$ grains comprise the model thickness ( $z$-direction). This combination of grain and primary crack geometry represents an L-T configuration (see Fig. 1). Each grain has thickness $t=0.1 \mathrm{~mm}$, representative of the flattened, pancake-shaped grains in rolled Al-Li plates. There are five alternating-orientation grains through the thickness of the model, for a total model thickness of $B=0.5 \mathrm{~mm}$.

In the $z$-direction, the boundary conditions impose zero out-of-plane displacements on just the top and bottom faces of the model (see Fig. 2). This allows significant, local variations in the $z$-direction across grains and GBs while approximating the macroscale near-plane-strain conditions ahead of a primary crack front at the centerplane of fracture specimen with zero $T$-stress.

The SSY framework applies plane-strain displacements representing Mode I fracture to the far boundary of the model in the linear-elastic region [32]. Here the boundary radius is $R=500 \mathrm{~mm}$. Boundary displacements are imposed in 500 equal-size steps to achieve a total $K_{\mathrm{I}}=47.5 \mathrm{MPa} \sqrt{\mathrm{m}}\left(J_{\mathrm{I}}=25.5 \mathrm{~kJ} / \mathrm{m}^{2}\right)$, representative of the loading intensity required to grow the primary crack in a divider configuration specimen of 2099-T87 Al-Li at cryogenic temperatures [18]. The maximum size of the plastic zone ahead of the primary crack is approximately $5 \mathrm{~mm}$. Macroscopically, this alloy yields at approximately $450 \mathrm{MPa}$ and has an ultimate tensile strength of about $550 \mathrm{MPa}$.

The boundaries between the soft/stiff grain pairs develop steep strain gradients; thus the mesh design biases most of the through-thickness refinement to the four interior GBs and the outside faces. The in-plane mesh refinement is sufficient to capture the effects of crack front blunting and the stress/strain gradients produced near the primary crack.

\subsection{Crystal plasticity material models}

The model described in Section 2.2 resolves fields and their gradients within individual grains having thickness on the order of $100 \mu \mathrm{m}$, with important regions near grain boundaries having additional resolution. At this length-scale, the deformation of metals, even single-phase alloy systems, becomes anisotropic with each grain having a different orientation, or rotation, relative to a common frame. Because the crystal systems of each grain are not aligned, each grain may have different effective properties in both elastic and plastic deformation. Single crystals of aluminum have nearly isotropic elastic properties; here the elastic deformation of all grains is considered isotropic. Anisotropy develops only from the effect of orientation on the plastic deformation of the material.

Plastic deformation in aluminum develops through the motion of dislocations along the 12 FCC slip systems - thus restricting plastic deformation within a single grain to simple shear in a combination of those 12 directions. Since the grains, in general, have different slip-system orientations due to their different crystallographic rotations, plastic deformation will not only be anisotropic in each single crystal, but 


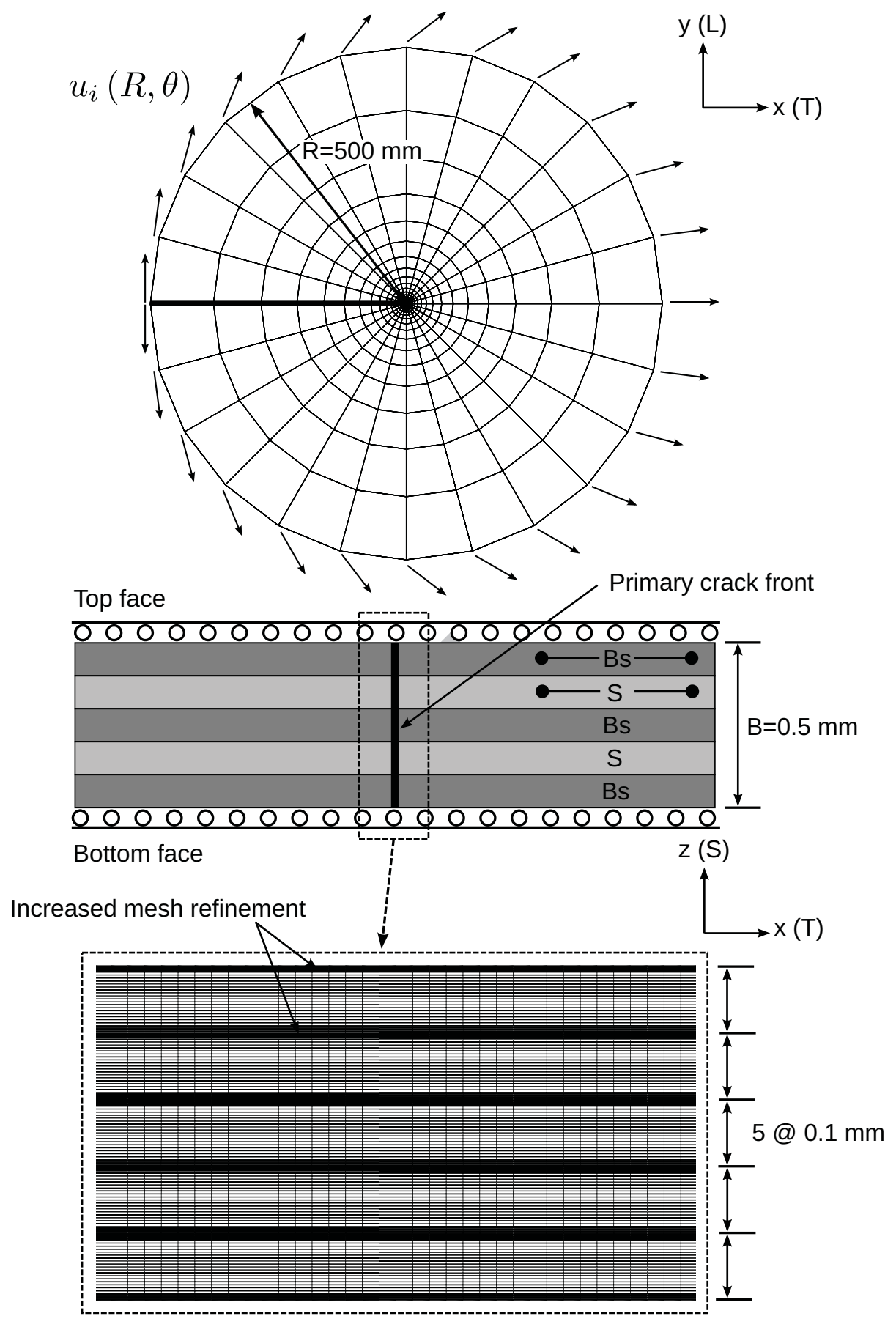

Figure 2: Small-scale yielding Mode I model of divider delamination. Model contains 600,000 nodes and 600,000 linear hexagonal elements with $\overline{\mathrm{B}}$ modification. Load ( $K$-field displacements) applied in 500 equal steps. Inner region near the primary crack front modeled with crystal plasticity; the region outside the plastic zone modeled as linear elastic. 
inhomogeneous across the model. The anisotropy and inhomogeneity lead to localized stress build-up along grain boundaries.

In a continuum setting, crystal plasticity represents the effect of orientation on mesoscopic plastic and elastic deformation. The flow rule derives from physical arguments for the motion of dislocations along a finite number of slip systems. For a multiplicative decomposition, $\mathbf{F}=\mathbf{F}^{e} \mathbf{F}^{p}$, Asaro [24] postulates the flow rule:

$$
\dot{\mathbf{F}}^{p} \mathbf{F}^{p-1}=\sum_{i=1}^{n_{s l i p}} \dot{\gamma}^{(s)}\left(\mathbf{b}^{(s)} \otimes \mathbf{n}^{(s)}\right)
$$

where $\dot{\gamma}^{(s)}$ is the slip rate, $\overline{\mathbf{b}}^{(s)}$ is the slip direction and $\overline{\mathbf{n}}^{(s)}$ is the slip normal for system $s$. Typically the slip rate $\dot{\gamma}^{(s)}$ is a power-law function of the resolved shear stress on the slip system, a slip system strength or backstress, and a reference strain rate:

$$
\dot{\gamma}^{(s)}=\frac{\dot{\gamma}_{o}}{\tilde{\tau}}\left|\frac{\tau^{(s)}}{\tilde{\tau}}\right|^{n-1} \tau^{(s)}
$$

Here $\tau^{(s)}$ is the resolved shear on system $s$, neglecting the kinematic effect of the small elastic stretch:

$$
\tau^{(s)}=\boldsymbol{\tau}:\left[\mathbf{R}^{e}\left(\mathbf{b}^{(s)} \otimes \mathbf{n}^{(s)}\right)\left(\mathbf{R}^{e}\right)^{T}\right]
$$

with $\mathbf{R}^{e}$ the rotational portion of the elastic deformation $\mathbf{F}^{e}, \boldsymbol{\tau}$ the Kirchhoff stress, $\dot{\gamma}_{o}=\sqrt{\frac{2}{3} \mathbf{D}: \mathbf{D}}$ with D the macroscale rate of deformation tensor, $\tilde{\tau}$ the slip system strength (here assuming all slip systems at a material point have the same strength), and $n$ a rate sensitivity constant, typically and here $n=20$. Appendix A provides details of the calculation of $\tilde{\tau}$ and material properties adopted in the simulations.

Previous simulations of fracture along a bicrystal interface demonstrate that including gradient plasticity via geometric hardening improves the fidelity of $\mathrm{CP}$ models when compared to the behavior observed in experiments $[33,34]$. Geometric hardening introduces a material length-scale, which may be particularly important when modeling grain boundary separation.

The Appendix describes two types of slip system hardening: (1) conventional work hardening due to the interaction of glissile dislocations with static forest dislocations and (2) geometric hardening caused by necessary dislocations. Necessary dislocations are required to accommodate net compatibility with a potentially incompatible plastic deformation. They are also the dislocations quantifiable in the continuum sense using the Nye tensor of dislocation density [35, 36]. These dislocations provide obstacles to glissile dislocation motion and therefore contribute to slip system hardening.

A material model based on these assumptions and the hardening model described in the Appendix represents the mesoscale deformation of the five grains in the SSY model. The full SSY model and this CP material model have been implemented in WARP3D, an open-source, 3D finite element package with extensive parallel computation capabilities (http://code.google.com/p/warp3d/). The simulations complete in a few hours on a current 12 core machine. 


\section{Results}

For an isotropic, homogeneous material the CP model reproduces the conventional SSY solutions in Mode I (with symmetry about $y=0$ ) including finite strain effects with no variations over the model thickness. The outer-face constraints produce simple plane-strain behavior [37, 38]. However, the anisotropic behavior of the grains modeled with CP causes significant grain-to-grain variations, with indications of the local driving forces for delamination present near the grain boundaries. In these simulations, the geometry and loading are symmetric about the $y=0$ plane, but the crystal plasticity material response is not - the full $\mathrm{FE}$ model $(\theta=2 \pi)$ enables proper representation of the asymmetric material response. However, under the strongly symmetric boundary conditions imposed by the asymptotic Mode I crack front displacements, the computed near-front fields reveal approximate symmetry about the $y=0$ plane. Figures $3-6$ show values from the CP analysis along a line through the thickness (in the $z$-direction) just ahead of the primary crack front in the $x-y$ plane. This line passes through all five grains and all four GBs. The vertical dashed lines in the figures indicate the locations of the GBs along the line.

Figure 3 shows the normal stresses and the mean stress along this line with values peaking on two of the grain boundaries. The in-plane normal stresses $\sigma_{x x}$ and $\sigma_{y y}$ increase near the GB, with the stresses building in the Bs orientation grain and then (discontinuously) jumping on the GB to a lower magnitude in the $\mathrm{S}$ orientation gain. The through-thickness normal stress $\sigma_{z z}$ also increases on the GBs, but here the stress increase occurs for both the $\mathrm{S}$ and Bs orientations, with no jump on the interface. Elevated values of the normal stresses on the GB lead to increased mean stress $\sigma_{m}=\left(\sigma_{x x}+\sigma_{y y}+\sigma_{z z}\right) / 3$ on the interface. While not shown, the in-plane shear stress $\tau_{x y}$ also increases on the same grain boundaries that experience a mean stress elevation. Small magnitude $(\approx 35 \mathrm{MPa})$ out-of-plane shears also develop.

The two other grain boundaries reveal decreased stress levels. Here the in-plane normal stresses undergo a sharp decrease in magnitude, with most of the decrease occurring in the $\mathrm{S}$ grains. The normal stress $\sigma_{z z}$ also decreases, but continuously in both orientations. The mean stress consequently has a locally smaller value on these grain boundaries. Significantly, stress increases occur in Bs orientation grains and decreases occur in S orientation grains; the Bs orientation grains have overall higher average stresses. However, most of the stress difference between the two orientations develops very near the GB.

Figure 4 highlights an important characteristic of the Bs and S orientations. This figure shows the increment of equivalent plastic strain $\Delta \bar{\varepsilon}^{p}=\sqrt{\frac{2}{3} \Delta \varepsilon_{i j}^{p} \Delta \varepsilon_{i j}^{p}}$ over the final increment of applied loading: $K_{\mathrm{I}}=47.4 \mathrm{MPa} \sqrt{\mathrm{m}} \rightarrow 47.5 \mathrm{MPa} \sqrt{\mathrm{m}}$. The $\mathrm{S}$ orientations undergo large plastic deformations, while the Bs orientations deform almost elastically. Since the Bs orientations deform with very little plasticity and, recalling Fig. 3, they generally deform at higher stress levels, particularly near the grain boundary, these orientations are termed stiff. In contrast, S orientation grains deform with significant plasticity and at lower stress levels near the GBs, these orientations are termed soft.

The Rice-Tracey parameter [39] is adopted here to provide an approximate characterization of pointwise 

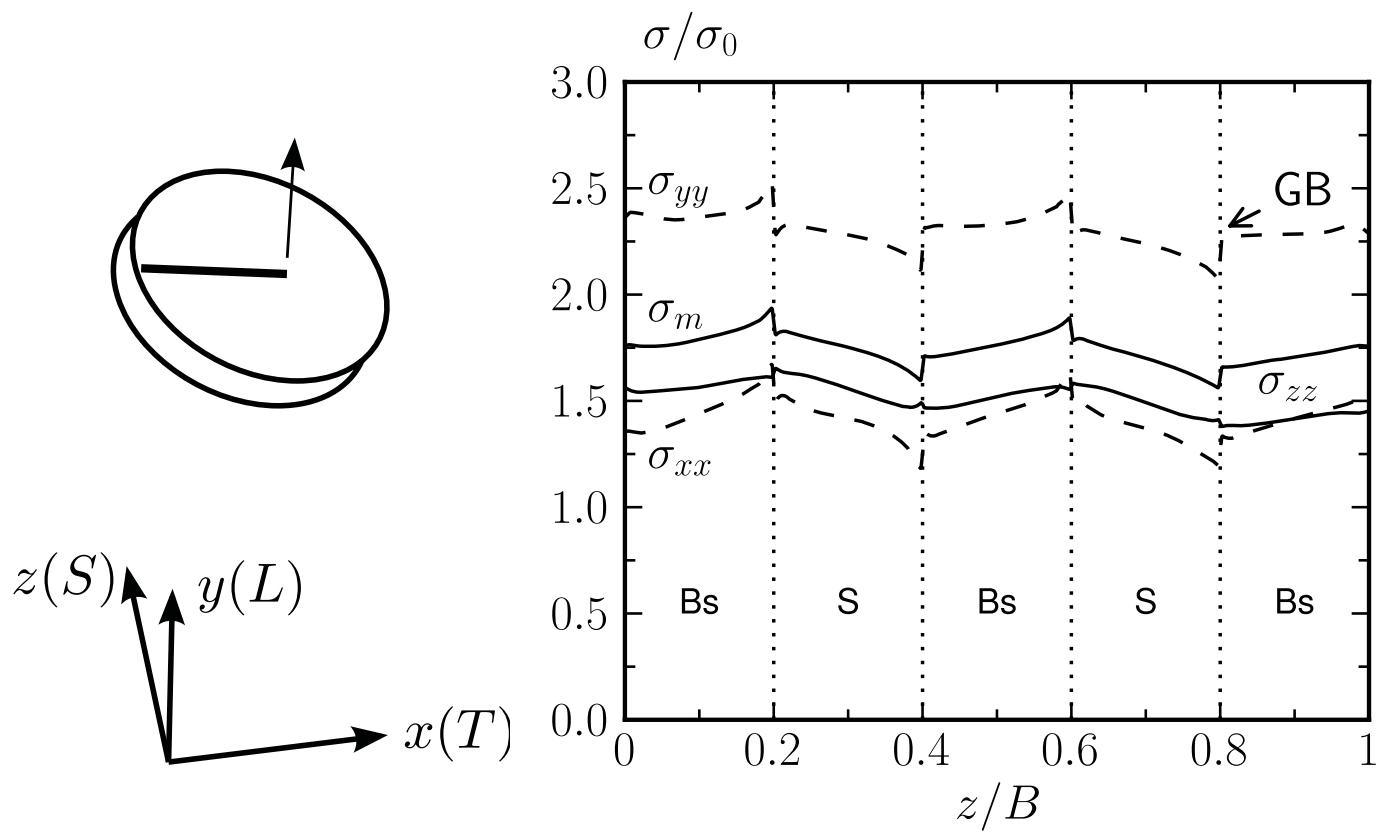

Figure 3: The normal stresses $\sigma_{x x}, \sigma_{y y}$, and $\sigma_{z z}$ and the mean stress $\sigma_{m}$, all normalized by the approximate, macroscopic yield stress $\sigma_{0}=445 \mathrm{MPa}$. The plot line is along the $z$-direction, passing through all 5 grains, at a point in the $x y$ plane $25 \mu \mathrm{m}$ ahead of the primary crack (see diagram on left). Here, $\sigma_{y y}$ is the opening mode stress on the primary crack.

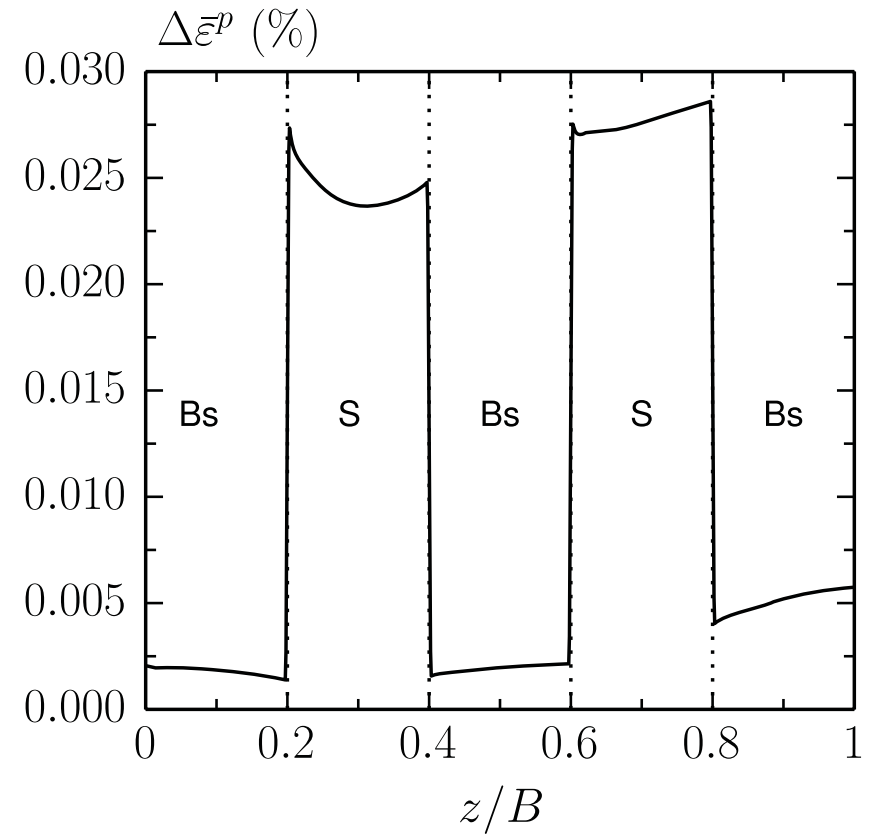

Figure 4: The increment of equivalent plastic strain $\bar{\varepsilon}^{p}=\sqrt{\frac{2}{3} \varepsilon_{i j}^{p} \varepsilon_{i j}^{p}}$ over the final step of applied loading, through the thickness of the model, along a line $25 \mu \mathrm{m}$ ahead of the primary crack front (see Fig. 3). The Bs orientations deform elastically, the S orientations deform plastically. 


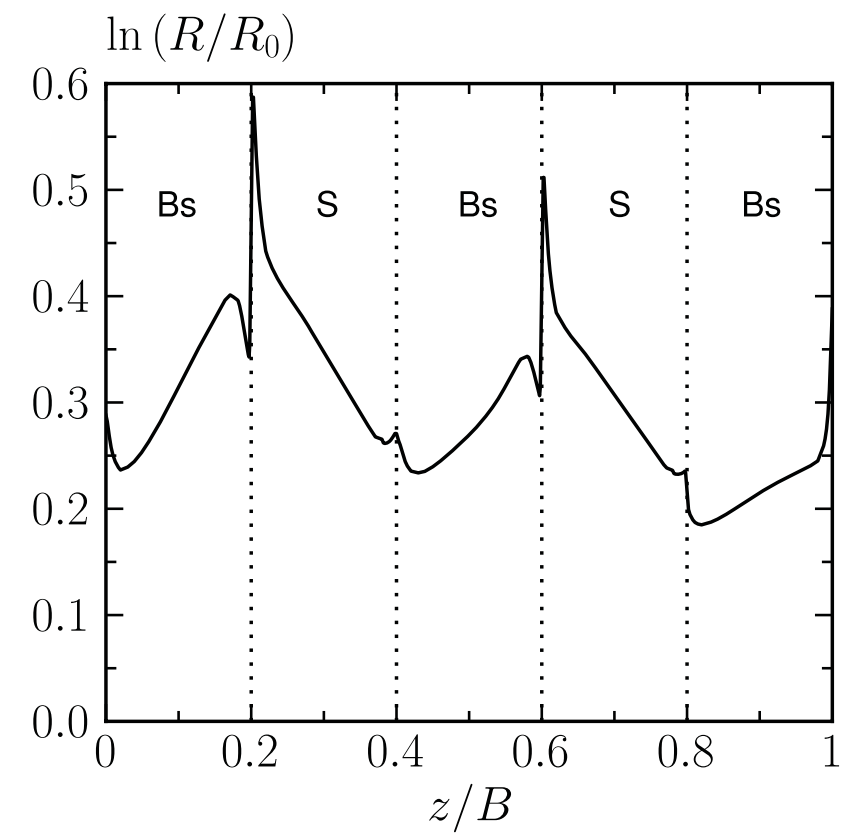

Figure 5: The Rice-Tracey void growth parameter through the thickness of the model at peak loading, along a line $25 \mu \mathrm{m}$ ahead of the primary crack front. The void growth parameter localizes strongly at specific grain boundaries.

conditions for void growth (see also d'Escata and Devaux [40])

$$
\ln \left(\frac{\bar{R}}{R_{0}}\right)=0.283 \int_{0}^{\varepsilon_{e q}^{p}} \exp \left(\frac{1.5 \sigma_{m}}{\sigma_{e}}\right) d \varepsilon_{e q}^{p} .
$$

Larger values of the Rice-Tracey parameter indicate locations more favorable for void growth. While initially developed for isotropic materials undergoing perfect $J_{2}$ plasticity, the parameter considers the correct factors - stress triaxiality and plastic deformation - that drive plastic void growth in anisotropic single crystals [41]. Regions with relatively high values of the parameter, compared to other points in the model, are indicated to experience relatively more void growth - even though the Rice-Tracey parameter may not capture the exact mechanics of void growth in single crystals. Figure 5 shows the Rice-Tracey parameter ahead of the primary crack front over the model thickness. The combination of elevated mean stresses and large plastic strains on two grain boundaries produce sharp spikes in the Rice-Tracey parameter - essentially doubling the values.

Finally, Fig. 6 shows the net density of necessary dislocations:

$$
\rho=\|\boldsymbol{\alpha}\|_{F}=\sqrt{\sum_{i=1}^{3} \sum_{j=1}^{3} \alpha_{i j}^{2}} .
$$

This quantity indicates regions of high plastic strain incompatibility, where large densities of necessary dislocations develop to maintain net compatibility. Regions with high necessary dislocation densities develop large amounts of geometric hardening, as these necessary dislocations oppose slip. The $\mathrm{S}$ orientations have 


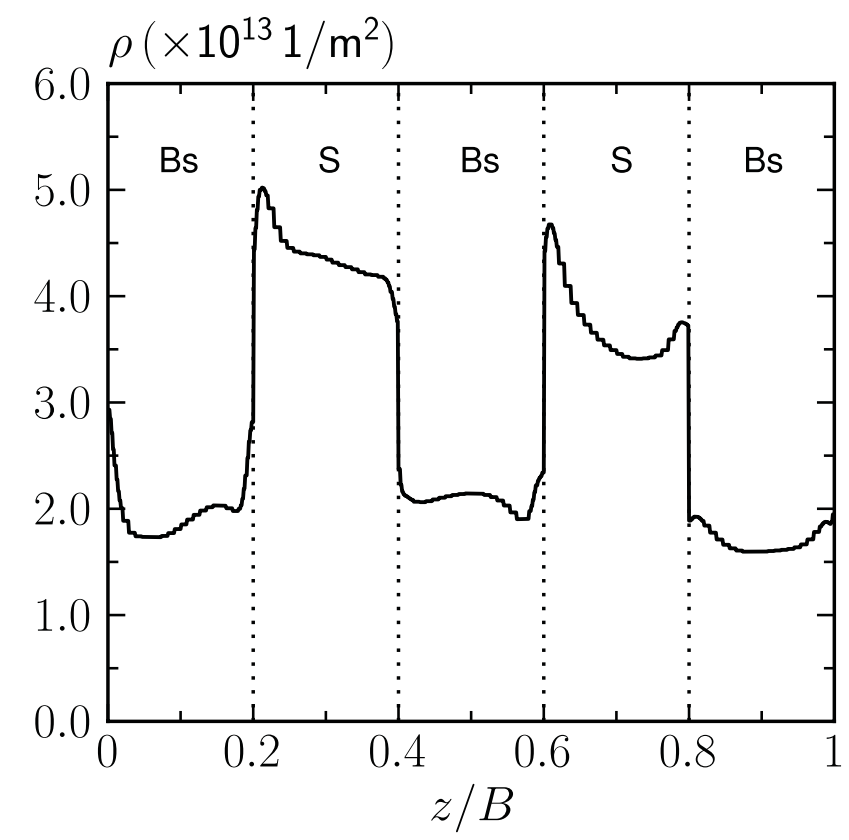

Figure 6: The necessary (net) dislocation density $\rho=\|\boldsymbol{\alpha}\|_{F}$ (Eqn. 5) at peak loading through the thickness of the model, along a line $25 \mu \mathrm{m}$ ahead of the primary crack front. Dislocations accumulate along grain boundaries, inducing further stress elevation through geometric hardening. The numerical calculation of dislocation density is constant within each element, producing the "stair step" appearance of the curve.

generally high densities of necessary dislocations and the dislocation density peaks on the grain boundaries. On the line plotted here, the contribution of geometric hardening to flow stress remains small - on the order of a few percent. However, closer to the primary crack front the geometric hardening contribution becomes greater, reflecting the large population of necessary dislocations required to maintain compatibility near the blunting crack front.

\section{Discussion}

\subsection{Initiation of delamination cracks}

The CP model in this SSY framework shows a strong localization of plastic strain and mean stress on certain grain boundaries and thus high values of the Rice-Tracey parameter used here to characterize approximately the conditions for void nucleation and growth. The void-growth mechanism matches experimentally observed fracture morphologies indicating the growth and coalescence of voids on fracture surfaces created by delamination cracking [8]. The simulations also reveal an elevated out-of-plane normal stress $\sigma_{z z}$. This elevated normal stress provides a potential microscale Mode I driving force to propagate newly-formed GB divider delaminations.

Experimental efforts to explain delamination cracking often focus on the combination of two effects grain boundary precipitates acting as trigger sites to nucleate voids and/or soft precipitate-free zones (PFZs) that drive localization of strain at the GBs [42, 43]. Figure 5 indicates that PFZs may not be critical to 
setting up conditions for delamination, as stress localizes at the grain boundaries in the present CP model that does not include PFZs (for example by lowering material stiffness of the GB regions). GBs in the current model have zero thickness - there is no boundary process zone. The compatibility/equilibrium mechanism proposed here may explain the ductile, intergranular failure observed by Decreus et al. [44] in AA2198. Decreus et al. do not observe PFZs in the alloy, but do note a post-failure surface consisting of fine dimples surrounding very small particles with diameters on the order of $50 \mathrm{~nm}$. Such a failure surface may arise from the growth and coalescence of voids, as described in this work, initially nucleated around the GB precipitates observed by Decreus et al.

The elevated mean stress on two of the GBs drives the RT parameter. This mean stress increase, caused by an increase in all three normal stress components, occurs in the stiff, Bs grains. The prediction of a stiff Bs grain that deforms elastically with high stresses adjacent to a soft $\mathrm{S}$ grain, deforming plastically at a lower stress level, agrees with experimental observations. As Fig. 3 shows, stress increases/decreases occur relatively near the GB, rather than more uniformly across the grain bulk. This agrees with the nanoindentation measurements in [31], which found a soft/stiff grain character only near the boundary. Figure 4 indicates the distinctly different deformation modes in the Bs and $\mathrm{S}$ grains $-\mathrm{S}$ grains deforming plastically, while Bs grains deform elastically. Figure 5 indicates the RT parameter peaking on the grain boundary and in the S grain, while the stress peaks on the GB and in the Bs grain - void growth remains low in the Bs grains because they do not develop the required plasticity. Overall, the model results indicate the maximum void growth occurs on the GBs where elevated mean stress and large plastic deformations occur simultaneously.

The in-plane shear elevation could also increase separation on the grain boundaries if the deviatoric part of the stress and plastic strain tensors contribute via a shear decohesion mechanism, as described by Bao and Wierzbicki [45] and Nahshon and Hutchinson [46]. However, the very triaxial stress state developed near the GBs in the simulation suggests a damage parameter considering only the diliational parts of the stress and strain, such as the Rice-Tracey parameter, sufficiently captures the mechanics of void growth indicating GB failure.

In $\mathrm{Al}-\mathrm{Li}$, the Bs grains respond more stiffly compared to the $\mathrm{S}$ grains solely from the direct effect of lattice orientation. The elastic and plastic hardening characteristics of Al-Li are nearly homogeneous across individual grains of all orientations. However, the 12 FCC slip systems impose limits on the kinematics of plastic deformation - grains with slip systems favorably aligned to the applied loading undergo more plastic deformation than grains with slip systems not favorably aligned. Our prior study [26] indicates the same result holds true for L-S orientations, and the experimental similarity of delamination in arrestor (L-S) and the present divider (L-T) configurations suggests that these orientations likely deform with a similar soft/stiff character under other delamination-prone loading configurations.

The simplest model of delamination cracking must then account for orientation of the material relative to the loading, or at least the soft/stiff response of the orientations under the applied loading. Characteristics 
that may have a secondary effect on delamination include the length-scale of the grains, particularly as this impacts the necessary dislocation density, geometric hardening, and the overall grain shape distribution of the sample. In particular, the pancake shape of grains in rolled Al-Li plates governs the growth of delamination cracks after initiation.

The present SSY model contains four soft/stiff, Bs/S GBs, but a mean stress increase occurs at only two of the four interfaces. On the other two GBs, the mean stress decreases - caused by a discontinuous decrease in the in-plane normal stresses in the $\mathrm{S}$ grains coupled with a continuous decrease of the out-of-plane normal stress in both grains. This stress decrease creates a local minimum in the RT parameter near these two GBs. Here, the CP model results do not indicate directly why the mean stress increases or decreases on the boundaries. A simple model, described in the next section, neglects details of the constitutive response and focuses on the interface compatibility/equilibrium requirements. This simple model explains why both a mean stress increase and a decrease are possible grain boundary responses.

\subsection{Elastic interface model}

Experimental observations and the $\mathrm{CP}$ model results presented above, particularly Fig. 4, indicate that soft/stiff deformation of the S/Bs grains defines the essential characteristic of the material response near the GBs. To further explore this result, with the eventual goal of a simplified model for engineering-scale computations, this section describes a simple two-layer, laminar model (see Fig. 7). The model comprises the linearized or tangent response of a single grain-pair composed of one stiff (Bs) and one soft (S) grain. After

linearization, both grains have a linear-elastic constitutive behavior with $E^{(1)}$ and $E^{(2)}$ and with the same $\nu$ value. The elastically-deforming Bs grain material has a high stiffness; the S grain has a much smaller stiffness. The grain boundary in this model (as in our CP simulation) has zero thickness. Plane-strain conditions imposed in the $n$-direction (see Fig. 7) approximate the conditions for a GB near a primary crack front in a fracture specimen/component at locations away from traction-free surfaces. Further, the large size of pancake-shaped grains in Al-Li relative to the thickness supports neglecting the in-plane $(s, t)$ dimensions in the laminar model.

The laminar model is loaded by macroscale, in-plane stresses denoted $\sigma_{s s}, \sigma_{t t}$, and $\sigma_{s t}$ determined from a smeared-type of higher-scale model that does not consider individual grains and grain boundaries. The goal here is then to determine microscale stress-strain values at the grain boundary for a range of $E^{(1)} / E^{(2)}$ driven by equilibrium and compatibility conditions.

As $t_{g}^{(1)}, t_{g}^{(2)} \rightarrow 0$, the material state on each side of the GB simplifies to one that must satisfy the conditions of interface compatibility and equilibrium. Here $\varepsilon^{(1)}, \sigma^{(1)}$ denote the strain/stress fields in grain 1 (macroscale plus changes caused by mismatches at the GB) and $\varepsilon^{(2)}, \boldsymbol{\sigma}^{(2)}$ the fields in grain 2 . The superscript $\mu$ indicates microscale fields and symbols without superscripts represent macroscale quantities.

With the boundary-orthogonal coordinate system $(n, s, t)$ shown in Fig. 7 , the fields may be written as:

$$
\varepsilon^{(1)}=\left[\begin{array}{llllll}
\varepsilon_{s s}^{\mu} & \varepsilon_{t t}^{\mu} & \varepsilon_{n n}^{\mu}+\frac{1}{2} \Delta \varepsilon_{n n}^{\mu} & \varepsilon_{t n}^{\mu}+\frac{1}{2} \Delta^{\mu} \varepsilon_{t n} & \varepsilon_{s n}^{\mu}+\frac{1}{2} \Delta^{\mu} \varepsilon_{s n} & \varepsilon_{s t}^{\mu}
\end{array}\right]_{1 \times 6}
$$




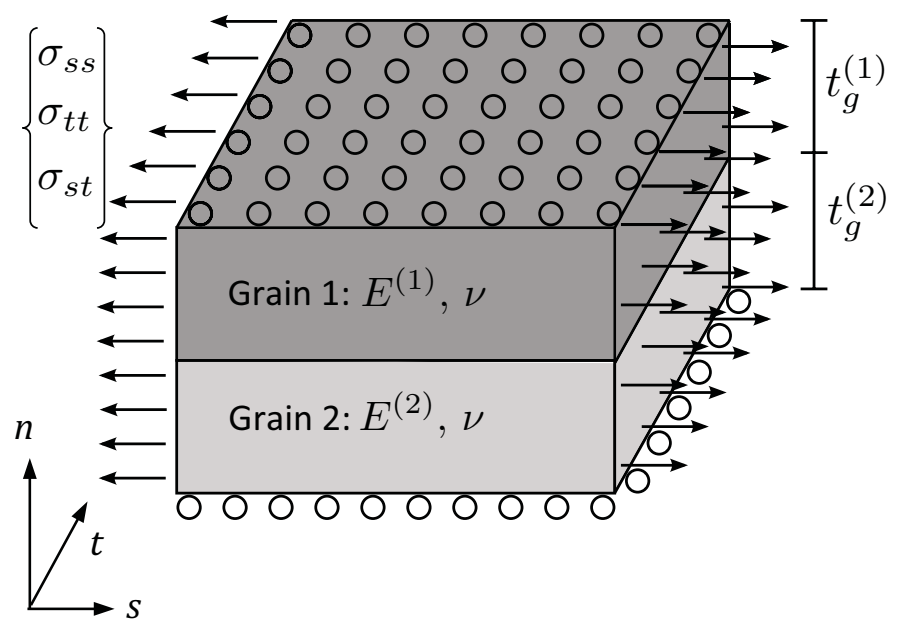

Figure 7: Simplified laminar model at a grain boundary. Note that the coordinate system here $(s, t, n)$ is different than used in the simulations. Here, the thin dimension of the grains is in the normal $(n)$ direction, and the wide grain dimensions are in the transverse directions $t$ and $s$. Rollers indicated on the $\pm n$ surfaces impose plane-strain conditions.

$$
\begin{gathered}
\varepsilon^{(2)}=\left[\begin{array}{ccccccc}
\varepsilon_{s s}^{\mu} & \varepsilon_{t t}^{\mu} & \varepsilon_{n n}^{\mu}-\frac{1}{2} \Delta \varepsilon_{n n}^{\mu} & \varepsilon_{t n}^{\mu}-\frac{1}{2} \Delta \varepsilon_{t n}^{\mu} & \varepsilon_{s n}^{\mu}-\frac{1}{2} \Delta \varepsilon_{s n}^{\mu} & \varepsilon_{s t}^{\mu}
\end{array}\right]_{1 \times 6} \\
\boldsymbol{\sigma}^{(1)}=\left[\begin{array}{cccccc}
\sigma_{s s}^{\mu}+\Delta \sigma_{s s}^{\mu} & \sigma_{t t}^{\mu}+\Delta \sigma_{t t}^{\mu} & \sigma_{n n}^{\mu} & \sigma_{t n}^{\mu} & \sigma_{s n}^{\mu} & \sigma_{s t}^{\mu}+\Delta \sigma_{s t}^{\mu}
\end{array}\right]_{1 \times 6} \\
\boldsymbol{\sigma}^{(2)}=\left[\begin{array}{llllll}
\sigma_{s s}^{\mu} & \sigma_{t t}^{\mu} & \sigma_{n n}^{\mu} & \sigma_{t n}^{\mu} & \sigma_{s n}^{\mu} & \sigma_{s t}^{\mu}
\end{array}\right]_{1 \times 6}
\end{gathered}
$$

with:

$$
\begin{aligned}
\boldsymbol{\varepsilon}^{(1)}-\boldsymbol{\varepsilon}^{(2)} & =\left[\begin{array}{lllllll}
0 & 0 & \Delta \varepsilon_{n n}^{\mu} & \Delta \varepsilon_{t n}^{\mu} & \Delta \varepsilon_{s n}^{\mu} & 0
\end{array}\right]_{1 \times 6} \\
\boldsymbol{\sigma}^{(1)}-\boldsymbol{\sigma}^{(2)} & =\left[\begin{array}{llllll}
\Delta \sigma_{s s}^{\mu} & \Delta \sigma_{t t}^{\mu} & 0 & 0 & 0 & \Delta \sigma_{s t}^{\mu}
\end{array}\right]_{1 \times 6} .
\end{aligned}
$$

In addition to satisfying the equilibrium and compatibility equations, the fields in each grain the satisfy linear-elastic constitutive equations:

$$
\begin{gathered}
\mathbf{C}^{(1)} \boldsymbol{\varepsilon}^{(1)}=\boldsymbol{\sigma}^{(1)} \\
\mathbf{C}^{(2)} \boldsymbol{\varepsilon}^{(2)}=\boldsymbol{\sigma}^{(2)} .
\end{gathered}
$$

We now set the in-plane components of microscale stress field equal to the macroscale, specified values to effectively load the laminar model:

$$
\begin{aligned}
\sigma_{s s}^{\mu} & =\sigma_{s s} \\
\sigma_{t t}^{\mu} & =\sigma_{t t} \\
\sigma_{s t}^{\mu} & =\sigma_{s t}
\end{aligned}
$$


and incorporate the local plane-strain conditions:

$$
\begin{aligned}
& \varepsilon_{n n}^{\mu}=0 \\
& \varepsilon_{t n}^{\mu}=0 \\
& \varepsilon_{s n}^{\mu}=0 .
\end{aligned}
$$

Under the plane-strain conditions, macroscopic $\sigma_{n n}=\nu\left(\sigma_{s s}+\sigma_{t t}\right)$. These conditions represent 18 linear equations: 6 each for the two linear-elastic constitutive relations, 3 for the in-plane stress constraint, and 3 for the local plane-strain conditions. There are 18 unknowns in the microscale stress/strain fields: 6 stress components, 6 strain components, 3 stress jumps, and 3 strains jumps. The constraints provide sufficient equations to solve for the unknown stress/strain fields in each grain: $\varepsilon^{(1)}, \boldsymbol{\sigma}^{(1)}$ and $\boldsymbol{\varepsilon}^{(2)}, \boldsymbol{\sigma}^{(2)}$.

In summary, the specified (macroscale) in-plane stresses drive the microscale response of this laminar model - which considers only an ideal, planar boundary, not the bulk response of the grain. The model considers marcoscale in-plane loading, though out-of-plane stress develops through the plane-strain constraint. The model is not suitable as a homogenization scheme to determine the total material response of a volume, as the bulk grain response then plays a significant role. However, in Al-Li the grain thickness is small compared to the dimension of the grains in the rolling plane with bulk response contributing less to the overall response.

Figure 8 shows key results from the laminar model for a range of $E^{(1)} / E^{(2)}$ : (1) the increase/decrease in mean stress relative to the specified macroscale value, and (2) the relative differences in normal stress $\sigma_{n n}$. Here $\Delta \sigma_{m}$ defines the mean stress increase/decrease in a hard grain over the macroscopic applied stress field, $\Delta \sigma_{m}=\Delta \sigma_{s s}^{\mu}+\Delta \sigma_{t t}^{\mu}+\left(\sigma_{n n}^{\mu}-\nu\left(\sigma_{s s}+\sigma_{t t}\right)\right)$, where $\sigma_{m}$ is the macroscopic mean stress $\sigma_{m}=$ $\sigma_{s s}+\sigma_{t t}+\nu\left(\sigma_{s s}+\sigma_{t t}\right)$. Similarly, $\Delta \sigma_{n n}=\sigma_{n n}^{\mu}-\nu\left(\sigma_{s s}+\sigma_{t t}\right)$ defines the difference between the microscale and macroscale stresses. Then:

$$
\begin{gathered}
\frac{\Delta \sigma_{m}}{\sigma_{m}}=\frac{(1-2 \nu)\left(E^{(1)}-E^{(2)}\right)\left[E^{(1)}+(1+\nu) E^{(2)}\right]}{2 \nu^{2} E^{(2)}\left(E^{(1)}-E^{(2)}\right)+(1-\nu) E^{(2)}\left(E^{(1)}+E^{(2)}\right)} \\
\frac{\Delta \sigma_{n n}}{\sigma_{n n}}=\frac{(1+\nu)(1-2 \nu)\left(E^{(1)}-E^{(2)}\right)}{2 \nu^{2}\left(E^{(1)}-E^{(2)}\right)+(1-\nu)\left(E^{(1)}+E^{(2)}\right)} .
\end{gathered}
$$

For $E^{(1)}>E^{(2)}$ a mean stress increase develops in grain 1 and an increase in the out-of-plane normal stress develops in both grains; for $E^{(1)}<E^{(2)}$ a mean stress decrease develops in grain 1 and a decrease in the out-of-plane normal stress develops in both grains. Both responses occur in the $\mathrm{CP}$ model on alternating grain boundaries (see Fig. 3). The ratio $E_{1} / E_{2}$ controls if a stress increase or decrease occurs; the Poisson's ratio only affects the magnitude of the increase or decrease.

Figure 9 illustrates graphically the response of this laminar model. Material elements on each side of the grain boundary must maintain strain compatibility under the constraint of the surrounding material. Further, the requirements of traction equilibrium across the interface limits the available mechanisms to 


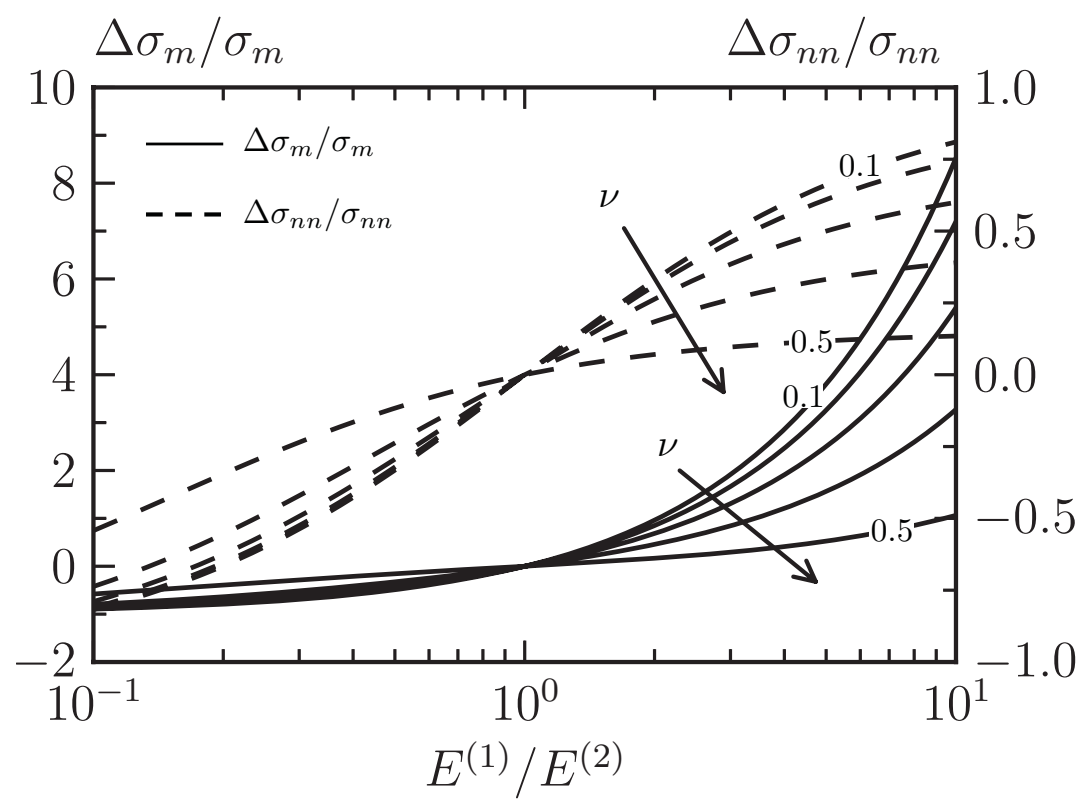

Figure 8: The mean stress and normal stress ratios from Eqns. 6 and 7 for various $\nu$ and ratios of $E^{(1)} / E^{(2)}$.

those outlined above and also found in the CP results (i.e. a mean stress increase or decrease in one grain, and a corresponding normal stress increase or decrease in both grains).

This laminar model thus reproduces key features of the response observed in the 3D CP simulation an interface between a soft material and a stiff material under confinement accommodates an applied inplane loading either with a mean stress increase in the stiff material or a mean stress decrease in the soft material. The visual description of deformation mechanisms shows intuitively why mean stress concentrates on Bs/S GBs, leading to the elevated indicators of void growth in the CP model. The laminar model does not reproduce the exact kinematics at the interface and greatly simplifies the consitutive response of the material to linear elasticity; consquently, it does not predict the magnitude of the GB stress increase or decrease. In the CP simulations, the stress increases/decreases are of approximate equal magnitude, whereas in the elastic laminar model the stress increases are larger than the decreases. However, the laminar model elucidates the mechanics affecting the mean stress on the soft/stiff boundaries.

Since both the CP simulation and the laminar interface model predict two possible responses for the mean stress under the specified in-plane loading, the question then arises how to predict which grain boundaries develop a stress increase and which develop a decrease. The answer for an individual GB will likely require refinement of this simple model to include effects of the grain bulk, surrounding grains, the influence of free surfaces, and the effect of the primary, transgranular crack. Each subvolume of material must obey equilibrium, implying that the microscale stresses must average to the global, macroscale stress. Therefore, for alternating configurations of soft/stiff grains, as in the simulations, the grains will alternate the stress 
Stress increase, $E^{(1)}>E^{(2)}$

Applied stress
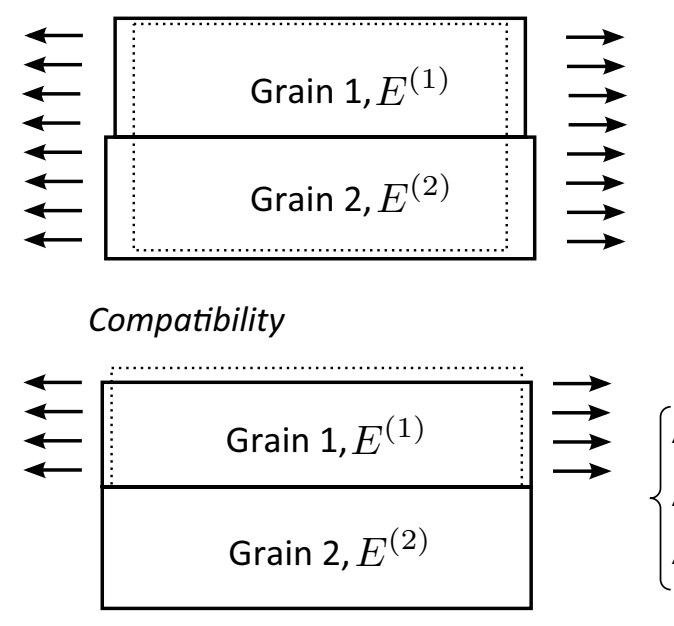

Constraint / Equilibrium

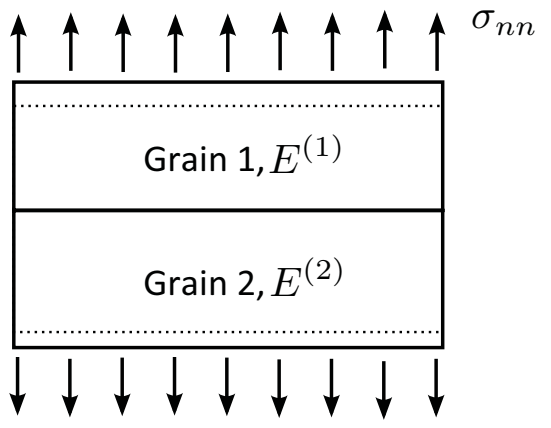

Stress decrease, $E^{(2)}>E^{(1)}$

Applied stress

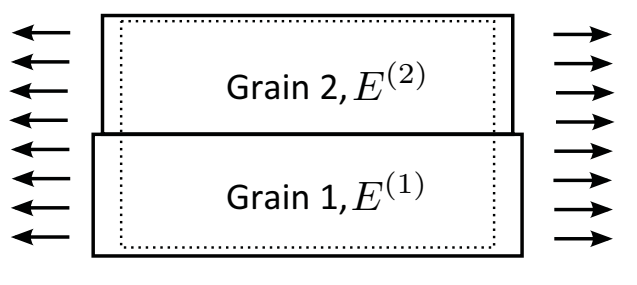

Compatibility

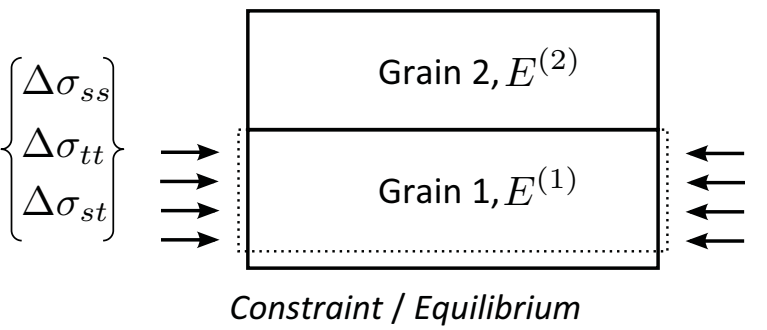

$\sigma_{n n}$

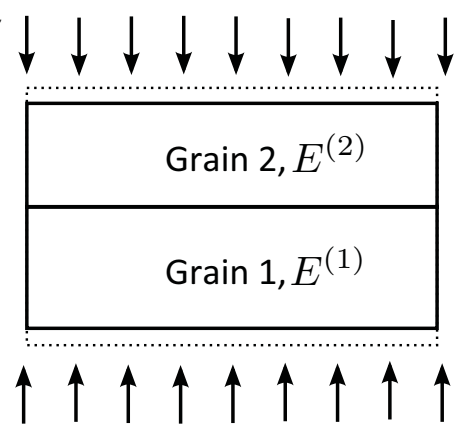

Figure 9: Illustration of loading/deformations in the simplifed laminar model. A stress increase occurs when the stiff grain accommodates the compatibility/equilibrium stresses, producing a mean stress increase in the stiff grain and an out-of-plane normal stress $\left(\sigma_{n n}\right)$ increase in both grains. A stress decrease occurs when the soft grain accommodates the compatibility/equilibrium stresses, producing a mean stress decrease in the soft grain and an out-of-plane stress decrease in both grains. 
increase/ stress decrease mechanisms to maintain equilibrium.

\subsection{Dislocation concentration and geometric hardening}

Figure 6 illustrates the accumulation of necessary dislocations on the soft/hard GBs. These dislocations are required to accommodate the incompatibility generated on the GB due to the differing soft/hard deformation modes found in each grain. They accumulate in regions of large plastic strains and especially in regions with a severe strain gradient - here at the GBs.

At the high strain rates ahead of propagating delamination cracks [21], void growth can occur by the emission of dislocation loops [47]. The density of necessary dislocations may also indicate void growth, supplementing use of the Rice-Tracey parameter. EBSD experiments can measure the excess dislocation density - an advantage over the Rice-Tracey parameter, which requires both stress and plastic strain data not commonly available from experiments.

\section{Summary and Conclusions}

This work describes a mechanism for the initiation of delamination cracks in Al-Li alloys based on the soft/stiff character of adjacent grains - observed in experiments to be delamination-prone. Small-scaleyielding, crystal plasticity simulations of divider grain configurations (L-T) reveal an elevated mean stress on grain boundaries. This mean stress increase drives a sharp localization of the Rice-Tracey parameter to the grain boundaries - elevation of the RT parameter indicates favorable conditions for void growth and triggering of delamination cracking, in agreement with the fractography of Ritchie and co-workers [7-14] Our simulation results and available experimental evidence indicate delamination initiates typically between soft/stiff grain pairs. The crystal plasticity results and a simple model of a soft/stiff material interface show that mean stress accumulation is a consequence of the mechanics of such an interface, and not necessarily tied to material inhomogeneities near the GBs (such as precipitate free zones). Delamination in Al-Li may then be considered quite similar to the separation of material layers in a soft-stiff laminar composite. Specific conclusions of this study are:

- Certain grain boundaries in crystal plasticity simulations of divider-configuration grains under SSY loading develop regions of increased void growth, as characterized by the Rice-Tracey parameter. This concentration indicates the localization of void nucleation and growth to the GBs, leading to grain boundary separation and delamination cracking.

- Elevated mean stress on GBs drives localization of the Rice-Tracey parameter. The mean stress localizes near the GB and has a magnitude of 20-30\% greater than the average/macroscopic mean stress over the model thickness. In particular, the in-plane normal stresses increase sharply near the GB in the Bs grain, with a discontinuous jump on the boundary itself, while the out-of-plane normal stress increases smoothly in both grains to reach an elevated value on the GB. 
- A simple laminar model of a single stiff/soft elastic interface predicts key features of the detailed crystal plasticity simulations.

- The initial applicability of this laminar model suggests that somewhat more advanced treatments may form the basis for a macroscale theory of delamination in Al-Li by incorporating the soft/stiff effect of mesoscale grain plasticity without detailed CP simulations of the crack-front region. In particular, the linear-elastic constitutive relations used here for the two grains will be replaced by a crystal plasticity material model. Such a local model may be capable of predicting the mesoscale stresses that trigger delamination within the framework engineering-scale analyses of structural components.

The next step to improve the two grain, laminar model of the grain boundaries will enforce the Hadamard compatibility conditions on the deformation gradient and the traction equilibrium conditions on the Cauchy stress:

$$
\begin{aligned}
& \llbracket \mathbf{F} \rrbracket=\mathbf{a} \otimes \mathbf{n} \\
& \llbracket \boldsymbol{\sigma} \rrbracket \cdot \mathbf{n}=\mathbf{0} .
\end{aligned}
$$

Evers et al. proposed such a full-interface model in the context of homogenization [48] - our ongoing work explores this approach with the grain response represented by the crystal plasticity material model.

\section{A. Crystal plasticity hardening model}

\section{A.1. Crystallographic orientation, rolling coordinates, and mesoscale plasticity}

The mesostructure of typical single phase metal alloys is a collection of crystalline grains, each with similar elastic and hardening properties but a different orientation of the atomic lattice. For materials produced by a rolling process, the orientation of each grain is typically measured with Euler angles defining the rotation from the grain crystal lattice to a standard set of orthogonal rolling coordinates: longitudinal $(\mathrm{L})$, in the direction of extrusion, short (S), along the dimension of reduction, and transverse $(\mathrm{T})$. In this work, we describe crystallographic orientations with Bunge convention Euler angles: a series of rotations $\left(\phi_{1}, \Phi, \phi_{2}\right)$, first about the $z$-axis, then about the $x$-axis, and finally again about the rotated $z$-axis.

The distribution of grain orientations defines the texture of a materal sample. For unrecrystallized, facecentered cubic metals the texture of a rolled sample tends to develop towards a set of standard orientations called a rolling texture. This rolling texture includes the copper, S, brass, Taylor, and Goss components each associated with a particular lattice orientation, which can be described by a set of Euler angles. Since all the grain orientations in a rolled FCC metal alloy tend to cluster around the orientations in this standard rolling texture, for convenience any orientation can be described by reference to a nearby component of the standard rolling texture. For example, a near-copper grain has a crystallgraphic orientation near the standard copper rolling component $-\phi_{1}=90^{\circ}, \Phi=35^{\circ}$, and $\phi_{2}=45^{\circ}$. 


\section{A.2. Crystal plasticity material model}

Section 2.3 describes the basic kinematics of crystal plasticity material models. That section does not provide the implementation of the slip system strength $\tilde{\tau}$ in Eqn. 2. This appendix outlines the details of the hardening model, based on the concept of mechanical threshold stress [18, 49] modified for geometric hardening.

All slip systems at a material point have the same strength, given as a function of the temperature $T$, strain rate $\dot{\varepsilon}=\sqrt{\frac{2}{3} \mathbf{D}: \mathbf{D}}$, and work hardening history of the point:

$$
\tilde{\tau}(t, T, \dot{\varepsilon})=\tau_{a}+\tau_{y}(T, \dot{\varepsilon}) \frac{\mu}{\mu_{0}}+\bar{\tau}\left(t, \tau_{v}(T, \dot{\varepsilon})\right) \frac{\mu}{\mu_{0}} .
$$

Here $\tau_{a}$ is a constant, athermal contribution, $\tau_{y}$ represents the slip resistance due to intrinsic barriers to dislocation motion, and $\bar{\tau}$ is the work hardening contribution - mediated by dislocation storage through mean free paths associated with geometric and statistical dislocation arrangements and first-order recovery. $\mu / \mu_{0}$ is the ratio between the current, temperature dependent shear modulus and the reference modulus. In the implementation $\mu(T)=\mu_{0}-D_{0} /\left[\exp \left(T_{0} / T\right)-1\right]$. The work hardening contribution obeys a modified Voce law:

$$
\frac{\partial \bar{\tau}}{\partial t}=\sum_{s=1}^{n_{\text {slip }}} \theta_{0}\left[1-\frac{\bar{\tau}}{\tau_{v}}+\frac{\tau_{\lambda}^{(s)}}{\bar{\tau}}\right]\left|\dot{\gamma}^{(s)}\right| .
$$

Neglecting the geometric hardening strength along each slip system $\tau_{\lambda}^{(s)}$, this expression is a linear Voce law with initial strength $\bar{\tau}(0)=\bar{\tau}_{i}>0$ and final strength $\tau_{v}$. The strengths $\tau_{y}$ and $\tau_{v}$ follow a MTS-type model for their rate and temperature dependence, with:

$$
\begin{aligned}
& \tau_{y}(T, \dot{\varepsilon})=\hat{\tau}_{y}\left\{1-\left[\frac{k T}{\mu(T) b^{3} g_{0, y}} \ln \left(\frac{\dot{\varepsilon}_{0, y}}{\dot{\varepsilon}}\right)\right]^{\frac{1}{q_{y}}}\right\}^{\frac{1}{p_{y}}} \\
& \tau_{v}(T, \dot{\varepsilon})=\hat{\tau}_{v}\left\{1-\left[\frac{k T}{\mu(T) b^{3} g_{0, v}} \ln \left(\frac{\dot{\varepsilon}_{0, v}}{\dot{\varepsilon}}\right)\right]^{\frac{1}{q_{v}}}\right\}^{\frac{1}{p_{v}}} .
\end{aligned}
$$

$\hat{\tau}$ is the mechanical threshold strength - the resistance to slip due to each effect with no energy provided by temperature or rate. $g_{0}$ is a normalized activation energy needed to overcome an obstacle, $p$ and $q$ are constants related to the shape of the energy barrier, $\dot{\varepsilon}_{o}$ is a reference strain rate, $b$ is the norm of the Burgers vector, and $k$ is the Boltzmann constant. Temperature and rate are related by an Arrhenius-type equation. In practice, $\hat{\tau}, g_{0}, p, q$, and $\dot{\varepsilon}_{0}$ are experimentally-determined constants, each must be determined for both intrinsic obstacles to dislocation motion, related to the yield stress of the material on the macroscale, and the forest hardening of the material, related to work hardening, on the macroscale.

Equation A.1 modifies the Voce law to account for the effect of necessary dislocations. The Nye tensor

$$
\boldsymbol{\alpha}=-\nabla \times \mathbf{F}^{e-1}
$$


quantifies the density of these necessary dislocations [35]. Following Acharya et al. [36, 50], the model finds the linear density of necessary dislocation along each slip system and apply their hardening effect to each system as:

$$
\tau_{\lambda}^{(s)}=k_{0} \frac{\eta^{2} \mu^{2} b}{2 \theta_{0}} \lambda_{n+1}^{(s)}
$$

where $\lambda^{(s)}=\sqrt{\left(\boldsymbol{\alpha} \overline{\mathbf{n}}^{(s)}\right):\left(\boldsymbol{\alpha} \overline{\mathbf{n}}^{(s)}\right)}$, the linear density of necessary dislocations along slip system $s$ with normal $\overline{\mathbf{n}}^{(s)}, \mu$ the shear modulus, $\eta \approx 1 / 3, b$ the norm of the Burgers vector, and $\theta_{0}$ the initial hardening slop from Eqn. A.1. $k_{0}$ is a dimensionless parameter which characterizes the geometric hardening response, determined experimentally (here $k_{0}=5$ ).

This hardening formulation, together with kinematic assumptions in Section 2.3, defines the CP material model. Table A.1 list the material properties used in the simulations. Properties for temperature and rate dependence are typical for Al-Li alloys [51]. These properties do not greatly affect the simulation in this study, as it is isothermal and the model is loaded at a relatively slow strain rate. The remaining properties fit the data from Kalyanam et al. - tension and compression tests of 2099-T87 Al-Li plate at cryogenic temperatures.

\section{Acknowledgements}

M. C. Messner's contribution to the paper was supported by the Department of Defense (DoD) through the National Defense Science \& Engineering Graduate Fellowship (NDSEG) Program. R. H. Dodds received support from the M.T. Geoffrey Yeh Chair Fund. A. J. Beaudoin received support through the US Department of Energy under Contract No. DEFG36-05GO15049. 


\begin{tabular}{cccc}
\hline Property & Description & Fit/typical & Value \\
\hline$E$ & Young's modulus & fit & $78811.2 \mathrm{MPa}$ \\
$\nu$ & Poisson's ratio & fit & 0.33 \\
$\mu=\mu_{0}$ & Shear modulus (isothermal) & fit & $29628.3 \mathrm{MPa}$ \\
$b$ & Burgers vector & typical & $3.5 \times 10^{-7} \mathrm{~mm}$ \\
$\tau_{a}$ & Athermal slip resistance & typical & $0 \mathrm{MPa}$ \\
$\hat{\tau}_{y}$ & MTS strength for intrinsic barriers & fit & $155 \mathrm{MPa}$ \\
$g_{0, y}$ & Normalized activation energy for intrinsic barriers & typical & $7.808 \times 10^{-3}$ \\
$\dot{\varepsilon}_{0, y}$ & Strain rate sensitivity for intrinsic barriers & typical & $1.0 \times 10^{13} 1 / \mathrm{s}$ \\
$q_{y}$ & Shape coefficient for intrinsic barriers & typical & 2.0 \\
$p_{y}$ & Shape coefficient for intrinsic barriers & typical & 0.5 \\
$\theta_{0}$ & Initial hardening slope & fit & $180 \mathrm{MPa}$ \\
$\hat{\tau}_{v}$ & MTS strength for forest hardening & fit & $25 \mathrm{MPa}$ \\
$g_{0, v}$ & Normalized activation energy for forest hardening & typical & $4.880 \times 10^{-3}$ \\
$\dot{\varepsilon}_{0, v}$ & Strain rate sensitivity for forest hardening & typical & $1.0 \times 10^{7} 1 / \mathrm{s}$ \\
$q_{v}$ & Shape coefficient for forest hardening & typical & 2.0 \\
$p_{v}$ & Shape coefficient for forest hardening & typical & 0.5 \\
$k_{0}$ & Geometric hardening parameter & typical & 5.0 \\
\hline
\end{tabular}

Table A.1: Material properties for CP simulations. 


\section{References}

[1] R. J. Rioja, J. Liu, The evolution of Al-Li base products for aerospace and space applications, Metall Mater Trans A 43 (2012) 3325-3337.

[2] J. J. Schubbe, Fatigue crack propagation in 7050-T7451 plate alloy, Engineering Fracture Mechanics 76 (2009) 1037-1048.

[3] R. D. Knutsen, R. Hutchings, Occurence of non-metallic inclusions in 3CR12 steel and their effect on impact toughness, Mater Sci Tech 4 (1988) 127-135.

[4] W. Guo, H. Dong, M. Lu, X. Zhao, The coupled effects of thickness and delamination on cracking resistance of X70 pipline steel, Int J Pres Ves Pip 79 (2002) 403-412.

[5] A. Csontos, E. Starke, The effect of processing and microstructure development on the slip and fracture behavior of the 2.1 wt pet Li AF/C-489 and 1.8 wt pet Li AF/C-458 Al-Li-Cu-X alloys, Metall Mater Trans A 31A (2000) 1965-1976.

[6] T. S. Srivatsan, T. Hoff, The high strain cyclic fatigue and fracture behavior of 2090 aluminum alloy, Eng Fract Mech 40 (1991) 297-309.

[7] K. T. Venkateswara Rao, R. J. Bucci, K. V. Jata, R. O. Ritchie, A comparison of fatigue-crack propogation behavior in sheet and plate aluminum-lithium alloys, Materials Science and Engineering A141 (1991) 39-48.

[8] K. T. Venkateswara Rao, R. O. Ritchie, Mechanical properties of Al-Li alloys Part 1 fracture toughness and microstructure, Materials Science and Technology 5 (1989) 882-895.

[9] K. T. Venkateswara Rao, R. O. Ritchie, Fatigue of aluminum-lithium alloys, International Materials Reviews 37 (1992) 153-185.

[10] K. T. Venkateswara Rao, W. Yu, R. O. Ritchie, Fatigue crack propogation in Aluminum-Lithium Alloy 2090: Part I. Long crack behavior, Metallurgical and Materials Transactions A 19A (1988) 549-561.

[11] K. T. Venkateswara Rao, W. Yu, R. O. Ritchie, Fatigue crack propogation in Aluminum-Lithium Alloy 2090: Part II. Small crack behavior, Metallurgical and Materials Transactions A 19A (1988) 563-569.

[12] K. T. Venkateswara Rao, W. Yu, R. O. Ritchie, On the behavior of small fatigue cracks in commercial aluminum-lithium alloys, Engineering Fracture Mechanics 31 (1988) 623-635.

[13] K. T. Venkateswara Rao, W. Yu, R. O. Ritchie, Cryogenic toughness of commercial Aluminum-Lithium alloys: Role of delamination toughening, Metallurgical Transactions A 20A (1989) 485-497.

[14] W. Yu, R. O. Ritchie, Fatigue crack propogation in 2090 Aluminum-Lithium alloy: effect of compression overload cycles, Journal of Engineering Materials and Technology 109 (1987) 81-85. 
[15] P. De, R. S. Mishra, J. A. Baumann, Characterization of high cycle fatigue behavior of a new generation aluminum lithium alloy, Acta Mater 59 (2011) 5946-5960.

[16] D. C. Vander Kooi, W. Park, M. R. Hilton, Characterization of cryogenic mechanical properties of Aluminum-Lithium alloy C-458, Scr Mater 41 (1999) 1185-1190.

[17] P. C. McKeighan, B. M. Hillberry, T. H. Sanders, Intergranular delamination and the role of artificial aging conditions on the fracture of an unrecrystallized Aluminum-Lithium-Zirconium (Al-Li-Zr) alloy, in: Fracture Mechanics: Twenty-Third Symposium, number 1189 in ASTM STP, pp. 515-535.

[18] S. Kalyanam, A. J. Beaudoin, R. H. Dodds, F. Barlat, Delamination cracking in advanced aluminumlithium alloys - Experimental and computational studies, Eng Fract Mech 76 (2009) 2174-2191.

[19] A. J. Beaudoin, M. Obstalecki, W. Tayon, M. Hernquist, R. Mudrock, P. Kenesei, U. Lienert, In situ assessment of lattice strain in an Al-Li alloy, Acta Mater 61 (2013) 3456-3464.

[20] W. Tayon, R. Crooks, M. Domack, J. Wagner, EBSD study of delamination fracture in Al-Li Alloy 2090, Exp Mech 50 (2010) 135-143.

[21] M. W. Hernquist, Effects of crack arresting delaminations in Aluminum-Lithium alloys, Master's thesis, University of Illinois at Urbana-Champaign, 2010.

[22] A. Needleman, Computational mechanics at the mesoscale, Acta Materialia (2000).

[23] F. Roters, P. Eisenlorh, L. Hantcherli, D. D. Tjahjanto, T. R. Biler, D. Raabe, Overview of constitutive laws, kinematics, homogenization, and muliscale methods in crystal plasticity finite-element modeling: theory experiments, applications, Acta Mater 58 (2010) 1152-1211.

[24] R. J. Asaro, Micromechanics of crystals and polycrystals, Adv Appl Mech 23 (1983) 1-106.

[25] R. J. McDonald, A. J. Beaudoin, Utilizing the meso-scale grain boundary stress to estimate the onset of delamination in 2099-T861 aluminum-lithium, Model Simul Mater Sc 18 (2010) 1-19.

[26] M. C. Messner, A. J. Beaudoin, R. H. Dodds, Jr., Mesoscopic modeling of crack arrestor delamination in Al-Li: Primary crack shielding and T-stress effect, International Journal of Fracture 188 (2014) 229-249.

[27] Y. Sugimura, P. G. Lim, C. F. Shih, S. Suresh, Fracture normal to a bimaterial interface: effects of plasticity on crack-tip shielding and amplification, Acta Metall Mater 43 (1995) 1157-1169.

[28] S. Roy Chowdhury, R. Narasimhan, A finite element analysis of quasistatic crack growth in a pressure sensitive constrained ductile layer, Engineering Fracture Mechanics 66 (2000) 551-571.

[29] G. Lin, Y.-J. Kim, A. Cornec, K.-H. Schwalbe, Fracture toughness of a constrained metal layer, Computational Materials Science 9 (1997) 36-47. 
[30] A. J. Beaudoin, M. Obstalecki, R. Storer, W. Tayon, J. Mach, P. Kenesi, U. Lienert, Validation of a crystal plasticity model using high energy diffraction microscopy, Modelling Simul Mater Sci Eng 20 (2012) 1-14.

[31] R. Crooks, M. S. Domack, J. A. Wagner, Microtexture and nanoindentation study of delamination cracking in Al-Cu-Li-X alloys, WIT Transactions on the Built Environment 85 (2006) 549-557.

[32] N. Levy, P. V. Marcal, W. J. Ostergren, J. R. Rice, Small scale yielding near a crack in plane strain: a finite element analysis, International Journal of Fracture Mechanics 7 (1971) 143-156.

[33] H. Tang, A. Acharya, S. Saigal, Directional dependence of crack growth along the interface of a bicrystal with symmetric tilt boundaries in the presence of gradient effects, Mechanics of Materials 37 (2005) 593-606.

[34] H. Tang, Y. S. Choi, A. Acharya, S. Saigal, Effects of lattice incompatibility-induced hardening on the fracture behavior of ductile single crystals, Journal of the Mechanics and Physics of Solids 52 (2004) $2841-2867$.

[35] M. F. Ashby, The deformation of plastically non-homogeneous materials, Philos Mag A 21 (1970) $399-424$.

[36] A. Acharya, A. J. Beaudoin, Grain-size effect in viscoplastic polycrystals at moderate strains, Journal of the Mechanics and Physics of Solids 48 (2000) 2213-2230.

[37] J. R. Rice, G. F. Rosengren, Plane strain deformation near a crack tip in a power-law hardening material, Journal of the Mechanics and Physics of Solids 16 (1968) 1-12.

[38] J. W. Hutchinson, Singular behavior at the end of a tensile crack in a hardening material, Journal of the Mechanics and Physics of Solids 16 (1968) 13-31.

[39] J. R. Rice, D. M. Tracey, On the ductile enlargement of voids in triaxial stress fields, Journal of the Mechanics and Physics of Solids 17 (1969) 201-217.

[40] Y. d'Escata, J. C. Devaux, Numerical study of the initiation, stable crack growth, and maximum load with a ducticle fracture criterion based on the growth of holes, in: ASTM STP 668, American Society of Testing and Materials, 1979, pp. 229-248.

[41] S. K. Yerra, C. Tekoglu, F. Scheyvaerts, L. Delannay, P. Van Houtte, T. Pardoen, Void growth and coalescence in single crystals, International Journal of Solids and Structures 47 (2010) 1016-1029.

[42] E. J. Lavernia, N. J. Grant, Review: Aluminum-Lithium alloys, Journal of Materials Science 22 (1987) $1521-1529$. 
[43] S. P. Lynch, B. C. Muddle, T. Pasang, Ductile-to-brittle fracture transitions in 8090 Al-Li alloys, Acta Mater 49 (2001) 2863-2874.

[44] B. Decreus, A. Deschamps, P. Donnadieu, J. C. Ehrström, On the role of microstructure in governing fracture behavior of an aluminum-copper-lithium alloy, Materials Science \& Engineering, A: Structural Materials: Properties, Microstructure and Processing 586 (2013) 418-427.

[45] Y. Bao, T. Wierzbicki, On fracture locus in the equivalent strain and stress tritriaxial space, International Journal of Mechanical Sciences 46 (2004) 81-98.

[46] K. Nahshon, J. W. Hutchinson, Modifications of the Gurson Model for shear failure, European Journal of Mechanics A 27 (2008) 1-17.

[47] V. A. Lubarda, M. S. Schneider, D. H. Kalantar, B. A. Remington, M. A. Meyers, Void growth by dislocation emission, Acta Materialia 52 (2004) 1397-1408.

[48] L. P. Evers, D. M. Parks, W. A. M. Brekelmans, M. G. D. Geers, Crystal plasticity model with enhanced hardening by geometrically necessary dislocation accumulation, Journal of the Mechanics and Physics of Solids 50 (2002) 2403-2424.

[49] U. F. Kocks, A. S. Argon, M. F. Ashby, Thermodynamics and kinetics of slip, in: B. Chalmers, J. W. Christian, T. B. Massalski (Eds.), Progress in Materials Science, volume 19, Pergamon Press, 1975.

[50] A. Acharya, J. L. Bassani, A. Beaudoin, Geometrically necessary dislocations, hardening, and a simple gradient theory of crystal plasticity, Scripta Materialia 48 (2003) 167-172.

[51] S. F. Hamel, A parametric study of delaminations in an Aluminum-Lithium alloy, Master's thesis, University of Illinois at Urbana-Champaign, 2010. 